\title{
The relationship between knee-ankle muscle strength and performance tests in young female adults with flexible pes planus
}

\author{
Serkan Bakırhan ${ }^{1 *}$ (D), Nuray Elibol ${ }^{1}$ (D), Mehmet Özkeskin ${ }^{1}$ (I) and Fatih Özden²
}

\begin{abstract}
Background: A decrease in muscular activation of the knee and ankle is one of the primary reasons for pes planus. The muscle strength of the knee and ankle in patients with pes planus may affect the performance tests which consists of daily life activities. The aim of the study was to determine the relationship between knee and ankle muscle strength with performance tests in young female adults with flexible pes planus. In addition, we also aimed to observe whether the severity of pes planus affects the ankle muscle strength and performance test scores. We carried out a cross-sectional single-center study. The muscle strength measurement of knee flexion-extension, ankle dorsal-plantar flexion, and ankle inversion-eversion movements were evaluated with the manual muscle test (MMT) device. The timed up and go test (TUG), 5-repeat sit-and-stand test (5STS), and 10-m walk test (10MWT) were used to evaluate knee and ankle performance.
\end{abstract}

Results: A significant difference was found only for the five-repetitive sit-and-stand test among the classified groups considering the pes planus grade $(p<0.05)$. There was no significant difference between groups for other parameters $(p>0.05)$. In correlation analysis, there was only a relationship between the timed up and go test (TUG) with left foot dorsal flexor muscle strength and between 10-m walk test (10MWT) with right foot invertor muscle strength $\left(r_{1}=-0.288, r_{2}=-0.288, p<0.05\right)$.

Conclusions: The results of this study reveal that the duration of 5STS was significantly lower in the second degree pes planus group. TUG test score showed low negative correlation with left foot dorsal flexor muscle strength. In addition, the scores of 10MWT exhibited poor negative correlation with right foot invertor muscle strength. Regarding other parameters, no significant correlations were found.

Keywords: Knee and ankle, Muscle strength, Performance tests, Pes planus

\footnotetext{
* Correspondence: bakirhan75@gmail.com

${ }^{1}$ Faculty of Health Sciences, Physiotherapy and Rehabilitation Department, Ege University, Ege Üniversitesi Sağlık Bilimleri Fakültesi 35100 Bornova, İzmir, Turkey

Full list of author information is available at the end of the article
}

(c) The Author(s). 2021 Open Access This article is licensed under a Creative Commons Attribution 4.0 International License which permits use, sharing, adaptation, distribution and reproduction in any medium or format, as long as you give appropriate credit to the original author(s) and the source, provide a link to the Creative Commons licence, and indicate if changes were made. The images or other third party material in this article are included in the article's Creative Commons licence, unless indicated otherwise in a credit line to the material. If material is not included in the article's Creative Commons licence and your intended use is not permitted by statutory regulation or exceeds the permitted use, you will need to obtain permission directly from the copyright holder. To view a copy of this licence, visit http://creativecommons.org/licenses/by/4.0/. 


\section{Background}

Pes planus (flatfoot) is one of the common orthopedic problems, usually caused by a decrease or disappearance of the medial longitudinal arch (MLA) of the foot [1]. Biomechanically, it has also been defined as the eversion or pronation of the heel accompanying the forefoot supination [2]. Apart from the changes in the hip, knee, and lumbar region biomechanics, bone, ligament, intrinsic, and extrinsic muscle strengths play an important role in the formation of pes planus due to the height of the MLA [3].

Knee and ankle muscle strength and control are associated with MLA height and is essential in functional activities and maintaining dynamic balance. The decrease or disappearance of MLA height due to pes planus causes a decrease in foot ground reaction forces, deterioration in balance parameters, gait disturbances, and a decrease in muscle strength endurance [4]. Studies on this issue have been reported to increase the trajectory of the foot to the pes planus due to the decrease in muscle strength in the knee and ankle, especially the tibialis posterior muscle $[5,6]$. Therefore, the decrease in muscular activation around the knee and ankle muscle girdle is important for the pes planus formation [6].

Performance-based physical evaluation tests are used in the functional evaluation of the patients. Physical performance tests are standardized tools that are used to monitor the patients during functional activities. Besides, these measurements provide objective quantitative scores (e.g., duration, number of repetitions) [7]. The timed up and go test (TUG), 5-repeat sit-and-stand test (5STS), and 10-m walk test (10MWT) are widely used in the clinical practice to measure lower extremity performance [8]. These tests contain the task related to the daily living activities of the patients. Performing these tests in patients with pes planus essential in terms of obtaining clinical information about their functional activities.

There is no study in the literature showing the relationship between knee and ankle muscle strength and performance tests in individuals with pes planus. The muscle strength of the muscles around the knee and ankle in patients with pes planus may affect their physical performance. This study aims to determine the relationship between knee and ankle muscle girdle strength and performance tests in patients with pes planus. We also aimed to observe whether the severity of pes planus affects the knee and ankle muscle strength and performance test scores.

\section{Methods}

\section{Subjects and procedures}

A cross-sectional study was carried out with 78 young female adults between December 2019 and August 2020 at Ege University Faculty of Health Sciences, Department of Nutrition and Dietetics, and Department of Midwifery. Measurement of the individuals with pes planus was carried out in the clinical laboratory of the faculty of health sciences. All muscle strength measurements and other performance tests were conducted by the same researcher (physiotherapist). The inclusion criteria were (1) individuals with flexible pes planus at the 1st, 2nd, and 3 rd level and $(2) \geq 18$ years. The exclusion criteria of the study were (1) a history of lower extremity surgery; (2) neurological, orthopedic, and vestibular impairments that can affect balance and gait; and (3) patients who do not give consent to participate in the study. The diagnosis of pes planus was carried out by Jack's test/toe rising test and Feiss-line by a physiotherapist who has clinical experience on pes planus.

In Jack's test, the physiotherapist raises the patient's toe passively upward and the formation of an arch is detected with the extensor hallucis longus mechanism. Jack's test is easy-to-use, common, and practical in diagnosing pes planus. Therefore, physiotherapists and physicians frequently use this test to diagnose pes planus [9]. Pes planus assessment was carried out while the patients were in a standing position with equally weightbearing to both extremities on a firm surface. In the standing position, normally, the tubercle of the navicular bone projects on the Feiss line which is drawn towards the center of the medial malleolus and metatarsophalangeal joint of the thumb.

The pes planus grades of the patients were evaluated according to the distance of the navicular tubercle from this line and towards the ground. If the navicular tubercle was located $1 / 3$ or $2 / 3$ down the distance between the Feiss line and the ground, pes planus was the 1st and 2nd grade, respectively. If the tubercule was completely touched the ground, the degree of the pes planus was 3rd [10]. The participants were grouped according to pes planus grades. There were 28, 26, and 24 participants in 1st, 2nd, and 3rd grade pes planus group, respectively.

The study was carried out in accordance with the ethical principles and the Helsinki Declaration. Informed consent of the patients was obtained. The study protocol was approved by the ethics committee of Ege University (No.: 19-7T/63). The study was funded by Ege University Scientific Project Research Office (No.: TGA-201920857).

\section{Outcome measurement tools}

\section{Knee and ankle muscle strength measurement}

Manual muscle test (MMT) device was used to measure knee and ankle muscle strength (Lafayette Hand Held Dynamometer, Model 01165). The MMT dynamometer was developed as a handheld device that detects the muscle strength of the patient, quantitively. MMT device 
is reliable, valid, and highly calibrated [11]. It has been widely used in many clinical trials. The measurement results could be obtained in kilograms or pounds. The device also measures the maximum power (peak force) and the time to reach the maximum power. It also has an automatic calibration feature. The measurement time can be set as $1-10 \mathrm{~s}$, at the end of this time it gives a signal that the user can hear, easily. In the evaluation of knee extensor muscle strength, the participant seated, while the hips and knees are $90^{\circ}$ flexed, the measurement was carried by placing a dynamometer on the proximal of the ankle joint (front of the shaft). For the knee flexor muscles, the knee and hip were at $90^{\circ}$ flexion while the participant was sitting. It was measured with a dynamometer placed at the back of the shaft, proximal to the ankle joint. Ankle plantar flexors are measured with the participant lying supine in hips and knees extended. In addition, invertor muscles were evaluated in the side-lying position. Dynamometer placed over the metatarsal heads on the sole of the foot. Ankle dorsiflexor muscles were evaluated with the participant lying supine with the ankle relaxed and also hips and knees extended. Evertor muscles were evaluated in the sidelying position. Dynamometer placed over the metatarsal heads on the dorsum of the foot. During the test, the "make test" technique, which requires isometric contraction, was used. While the evaluator kept the dynamometer constant, the person being measured applied maximum power against the device. Both of the measurement results were taken in kilograms $(\mathrm{kg})$. The measurements were repeated 3 times for each muscle group and the average values were recorded [12].

\section{The timed up and go test (TUG)}

The timed up and go test is a popular tool among clinicians to assess functional mobility. It includes many basic mobility components such as balance, transfer, walking, and turning. According to the testing protocol, the individual was seated in a standard chair. It was marked by measuring a distance of $3 \mathrm{~m}$ forward from the level of the chair. The participant, sitting on the chair with his back leaning, was asked to stand up and walk at a normal walking speed with the "Start" command, return from the 3-m mark and sit on the chair again. The time elapsed between the moment the patient lifts his back from the chair and the moment he comes back and rests his back was recorded. Measurements were repeated 3 times and the average duration was calculated as a patient score [13].

\section{Five-repeat sit-and-stand test (5STS)}

The participants were asked to stand up and sit as fast as possible 5 times on a standard chair $(42-45 \mathrm{~cm})$, with their hands on their shoulders, arms crossed on the chest. They were initiated to perform the test with the "Start" command, and the duration was recorded until the end of the 5th repetition. The time was stopped with the contact of the hip with the chair. Measurements were repeated 3 times and the average duration was calculated as a patient score [14].

\section{Ten-m walk test (10MWT)}

10MWT is a performance test in which walking is evaluated by recording the walking performance of the $10-\mathrm{m}$ distance of the patient. It is used to determine functional mobility, walking speed, and also vestibular functions. The duration of walking the $10-\mathrm{m}$ distance between the first $5 \mathrm{~m}$ given for acceleration and the last $5 \mathrm{~m}$ given for deceleration was recorded. Measurements were repeated 3 times and the average duration was recorded [15].

\section{Statistical analysis}

All statistical analysis was calculated by using SPSS for Windows v25.0 software (SPSS Inc., Chicago, IL, USA). For quantitative variables, mean and standard deviation (SD) were presented. Percent (\%) were reported for qualitative variables. The confidence interval of $95 \%$ was accepted. The normal distribution of data was analyzed by Shapiro-Wilk or Kolmogorov-Smirnov tests. The significant difference between the groups in terms of ankle muscle strength and performance tests was analyzed by one-way analysis of variance (ANOVA). The relationship between knee and ankle girdle muscle strength and performance tests were analyzed using the Pearson correlation coefficient $(r)$. The Pearson correlation coefficients were interpreted as $0-0.19=$ very low, $0.20-0.39=$ low, $0.40-0.69=$ medium, $0.70-0.89=$ high, and $0.90-1.0=$ very high [16].

In order to reduce type 2 errors, the sample size was determined by performing power analysis before the data collection period. The minimum adequate sample size calculated using $G^{*}$ power 3.1 software (effect size $=0.3$, an error probability of 0.05 , and power $=0.80$ ). Accordingly, 64 participants were required. We completed the study with 78 patients $[17,18]$.

\section{Results}

The mean age of the patients was $20.65 \pm 0.14$ years. The patients were grouped according to their pes planus levels. $35.9 \%$ was 1 st degree, $33.3 \%$ was 2 nd degree, and $30.8 \%$ was 3 rd degree pes planus. The physical characteristics of the individuals are given in Table 1. The absolute values of the dynamometer measurement and physical performance test results were given in Table 2 . The duration of the timed up and go test was $4.96 \mathrm{~s}$, the 5-repetitive sit and go test was $8.36 \mathrm{~s}$, and the $10-\mathrm{m}$ walking test time was $5.85 \mathrm{~s}$. As a result of the variance analysis, there was a significant difference between the 
Table 1 The physical characteristics of all participants and the groups

\begin{tabular}{lllll}
\hline $\boldsymbol{n}$ : 78 & Total & 1st grade & 2nd grade & 3rd grade \\
\hline Age (years, mean \pm SD) & $20.65 \pm 0.14$ & $20.60 \pm 1.47$ & $20.73 \pm 1.00$ & $20.62 \pm 1.27$ \\
Weight $(\mathrm{kg}$, mean \pm SD) & $56.64 \pm 7.85$ & $56.67 \pm 8.05$ & $53.84 \pm 5.64$ & $59.62 \pm 8.81$ \\
Height $(\mathrm{cm}$, mean $\pm \mathrm{SD})$ & $164.94 \pm 6.16$ & $165.46 \pm 5.70$ & $163.23 \pm 5.96$ & $166.20 \pm 6.71$ \\
$\mathrm{BMI}\left(\mathrm{kg} / \mathrm{m}^{2}\right.$, mean $\left.\pm \mathrm{SD}\right)$ & $20.79 \pm 2.49$ & $20.73 \pm 3.05$ & $20.20 \pm 1.70$ & $21.51 \pm 2.40$ \\
\hline
\end{tabular}

SD standard deviation, $n$ number of patients, $\mathrm{kg}$ kilogram, $\mathrm{cm}$ centimeter

groups only for the five-repetitive sit-and-stand test $(p<$ $0.05)$. Second degree pes planus group completed 5STS in a significantly shorter time than the third and first degree pes planus group. There was no significant difference between groups for other parameters $(p>0.05)$ (Table 2). There was a low significant negative correlation between TUG with left foot dorsal flexor muscle strength and between 10MWT with right foot invertor muscle strength $\left(r_{1}=-0.288, r_{2}=-0.255, p<0.05\right)$ (Table 3).

\section{Discussion}

In the present study, we aimed to demonstrate the relationship between knee and ankle muscle strength with performance tests in young female adults with flexible pes planus. Besides, we also aimed to observe whether the severity of pes planus affects the ankle muscle strength and performance test scores. Due to the collapse of the medial longitudinal arch of the foot in pes planus, related biomechanical problems could cause balance and posture disorders [19]. According to our results, we determined that muscle strength values are not related to performance test parameters. Also, pes planus severity does not affect the physical performance of the patients.

Due to the low arch heights, lower extremity injuries are more common in different degrees of pes planus than healthy individuals with normal arch height [20]. In a prospective study, it was reported that soft tissue and foot injuries were more common in individuals with high arch height compared to people with low arch height [21], while this injury caused by falls was reported to be related to foot muscle strength [22]. In our study, manual muscle strength (MMS) device was used to evaluate ankle muscle strength. This hand-held detects the resistance given by the person performing the test and objectively shows the amount of force used. The MMT device provides accurate measurement on a scientific basis [12]. Accordingly, the MMT device was used in our study, because it is easy to use, practical, and provides objective quantitative results. Studies examining the relationship between ankle muscle strength and arch height are quite limited in the literature. No other study was carried out with a manual muscle strength device. Zhao et al. examined the effect of different arch heights on ankle muscle strength. They investigated the muscle

Table 2 Absolute values of the measurements and one-way analysis of variance (ANOVA) between pes planus groups

\begin{tabular}{|c|c|c|c|c|c|c|}
\hline & Total & 1st degree & 2nd degree & 3rd degree & $F$ & $p$ \\
\hline Knee extensor-right & $9.73 \pm 1.81$ & $9.37 \pm 1.63$ & $9.22 \pm 1.37$ & $9.73 \pm 1.81$ & 5.647 & 0.052 \\
\hline Knee extensor-left & $9.70 \pm 1.79$ & $9.44 \pm 1.71$ & $9.39 \pm 1.44$ & $10.36 \pm 2.10$ & 2.379 & 0.100 \\
\hline Knee flexor-right & $11.16 \pm 1.51$ & $11.33 \pm 1.54$ & $10.66 \pm 1.11$ & $11.52 \pm 1.75$ & 2.390 & 0.099 \\
\hline Knee flexor-left & $10.68 \pm 1.41$ & $10.76 \pm 1.55$ & $10.31 \pm 0.96$ & $10.98 \pm 1.61$ & 1.513 & 0.227 \\
\hline Dorsiflexor-right & $10.74 \pm 1.91$ & $10.30 \pm 2.07$ & $10.88 \pm 1.94$ & $11.11 \pm 1.62$ & 1.256 & 0.291 \\
\hline Dorsiflexor-left & $10.44 \pm 1.98$ & $9.98 \pm 2.12$ & $10.63 \pm 2.14$ & $10.76 \pm 1.56$ & 1.206 & 0.305 \\
\hline Plantar flexor-right & $11.50 \pm 0.97$ & $11.47 \pm 0.93$ & $11.39 \pm 0.62$ & $11.67 \pm 1.30$ & 0.525 & 0.594 \\
\hline Plantar flexor-left & $11.92 \pm 1.21$ & $11.97 \pm 0.95$ & $11.74 \pm 1.26$ & $12.07 \pm 1.44$ & 0.489 & 0.615 \\
\hline İnvertor-right & $9.26 \pm 0.99$ & $9.43 \pm 1.00$ & $8.94 \pm 1.03$ & $9.42 \pm 0.87$ & 2.182 & 0.120 \\
\hline İnvertor-left & $9.26 \pm 1.10$ & $9.41 \pm 1.21$ & $8.92 \pm 0.98$ & $9.47 \pm 1.06$ & 1.973 & 0.146 \\
\hline Evertor-right & $9.63 \pm 1.02$ & $9.66 \pm 0.96$ & $9.28 \pm 1.00$ & $9.97 \pm 1.01$ & 3.028 & 0.054 \\
\hline Evertor-left & $9.27 \pm 1.00$ & $9.47 \pm 1.12$ & $9.01 \pm 0.84$ & $9.33 \pm 1.01$ & 1.485 & 0.233 \\
\hline TUG & $4.96 \pm 0.53$ & $4.98 \pm 0.58$ & $4.97 \pm 0.57$ & $4.93 \pm 0.44$ & 0.048 & 0.954 \\
\hline 5STS & $8.36 \pm 0.98$ & $8.66 \pm 1.14$ & $7.95 \pm 0.76$ & $8.44 \pm 0.89$ & 3.873 & $0.025^{*}$ \\
\hline 10MWT & $5.85 \pm 1.29$ & $5.88 \pm 1.41$ & $6.15 \pm 1.47$ & $5.49 \pm 0.79$ & 1.640 & 0.201 \\
\hline
\end{tabular}

TUG the timed up and go test, 5STS 5-repeat sit-and-stand test, 10MWT 10-m walk rest, $s$ second ${ }^{*} p<0.05$ 
Table 3 The relationship between the average knee-ankle muscle strength and performance test measurements

\begin{tabular}{llll}
\hline $\mathbf{n}$ : 78 & TUG & 5STS & 10MWT \\
\hline Knee extensor-Right & -.192 & .042 & -.007 \\
Knee extensor-Left & -.135 & .000 & .044 \\
Knee flexor-Right & -.188 & -.075 & -.171 \\
Knee flexor-Left & -.183 & -.086 & -.133 \\
Dorsiflexor-Right & -.181 & .138 & .142 \\
Dorsiflexor-Left & $-.288^{*}$ & .072 & .216 \\
Plantar flexor-Right & -.021 & .134 & -.051 \\
Plantar flexor-Left & -.073 & -.023 & .071 \\
İnvertor-Right & -.039 & .059 & $-.255^{*}$ \\
İnvertor-Left & -.014 & .098 & -.180 \\
Evertor-Right & -.071 & .029 & -.171 \\
Evertor-Left & -.023 & -.077 & -.129 \\
\hline
\end{tabular}

TUG the timed up and go test, 5STS 5-repeat sit-and-stand test, 10MWT 10-m walk test

${ }^{*} p<0.05$

strength values of the ankle dorsiflexor and plantar flexor at $30 \%$ and $120 \%$, and the ankle invertor and evertor muscle groups in 67 patients. They determined a negative correlation between high arch level and ankle muscle strength; they found that patients with low arch level had higher muscle strength values [23]. In another study, Aydoğ et al. found a negative correlation between arch height and ankle eversion muscle strength in a comparative study on 20 gymnastic athletes and 17 healthy and sedentary cases [24]. In our study, we examined the difference of arch height on knee and ankle muscle strength. There was no significant difference between ankle muscle strength and pes planus grades in young female adults aged 18-24. The lack of significant difference between the groups may be due to the fact that our patients are young individuals with high activity levels.

Balance is the ability to control the body against the body's center of gravity changes in static and dynamic positions with minimal muscle activity. In this respect, maintaining balance and keeping center of gravity on the support surface is provided by coordination between appropriate neural mechanisms and the musculoskeletal system [25]. Especially the ankle strategy has an essential place in maintaining balance. The ankle strategy maintains ground balance by shifting the center of gravity around the ankle more slowly. Therefore, ankle muscle strength is important in maintaining balance [22]. In individuals with pes planus, in addition to the inequality in the load distribution caused by the collapse of the medial arch support, the weakness of the muscle, tendon, and ligament structures, accompanied by biomechanical disorders, causes rapid fatigue and balance disorders in individuals. As a result, the duration of individuals with pes planus to stay in balance decreases, and balance parameters are negatively affected [19]. Telfer et al. reported that the support surface of the patients with pes planus is increased, but changes in the medial arch structure negatively affect walking ability [26]. In our study, we observed that there was no difference between the timed up and go test times used in the dynamic balance assessment of individuals with different degrees of pes planus. In addition, the muscle strength values in individuals with different pes planus degrees did not correlate with dynamic balance parameters. We thought that the similarity of age groups could be the reason for the similar dynamic balance parameters.

Performance tests are used to observe functionality. Among these tests, TUG is one of the important measurement tools that evaluate the individual's ability to stand up and sit, as well as walking ability, and show the independence level of the individual in daily life. The World Health Organization defines the failure in this activity as one of the most essential inadequacy of the patients in daily life. For this purpose, it is a frequently preferred test due to the simplicity of the use, which does not require special equipment and costs [7, 27]. Performance tests make important statements about the individual's overall sense of strength, agility, and proprioceptive. Each of these parameters is sensitive to biomechanical changes in the lower extremity [23]. Therefore, in our study, we used this sit-and-stand test to evaluate the relationship between knee extensor muscle strength and performance tests in individuals with pes planus. Lin et al. demonstrated that pes planus is associated with motor performance levels in preschool children [28]. In another study, Oda et al. reported that the degree of pes planus has no effect on motor functions [29].

In our study, patients with 2nd degree pes planus completed the 5-repeat sit-and-stand test in a shorter time than other pes planus degrees, although there was a slight but significant difference. We interpreted that this difference may not have affected physical performance due to pes planus, since the difference between the groups in the time to complete the 5-repeat sit-andstand test was less than one second. However, we think that it will be important to include a sample consist of different age groups in the study in order to better analyze the effect of arch height on the performance level, in order to give more objective results. Also, exclusion criteria (e.g., body mass index) could be more comprehensive. Besides more decisive and predictive performance tests (e.g., plyometric tests) might be more effective to reveal the precise results.

Walking is one of the important activities that determines the lower extremity performance level. The deformity in the foot due to the pes planus causes these individuals to have higher peak pressure and more 
contact areas due to more pressure on the forefoot [30]. In the related studies, the changing foot plantar pressure ultimately leads to changes in walking patterns, especially a decrease in walking speed [31, 32]. One of the tests evaluating walking speeds are the TUG and 10MWT. The 10-m walking test is a simple, reliable, valid, sensitive, and easy-to-use [15]. In correlation analysis, there was only a relationship between TUG with left foot dorsal flexor muscle strength and between $10 \mathrm{MWT}$ with right foot invertor muscle strength. We concluded that gait-based performance tests are associated with knee and ankle muscle strength. We commented that the 5-repetitive sitting and standing test might not have been associated since it did not require walking. For this reason, we used the TUG and 10MWT to evaluate walking in our study. In our study, there was no correlation between muscle strength and walking speed, while a relationship was determined only between the invertor muscles and 10MWT. It may be important to include individuals without pes planus in the study as a control group and to compare them with individuals with different degrees of pes planus.

\section{Conclusion}

The results of this study reveals that the duration of 5STS was significantly lower in the second degree pes planus group. TUG test score showed low negative correlation with left foot dorsal flexor muscle strength. In addition, the scores of 10MWT exhibited poor negative correlation with right foot invertor muscle strength. Regarding other parameters, no significant correlations were found.

\section{Abbreviations}

5STS: Five-repeat sit-and-stand test; 10MWT: Ten-m walk test; MLA: Medial longitudinal arch; MMT: Manual muscle test; TUG: The timed up and go test; SD: Standard deviation; $n$ : Number of patients; $r$ : Spearman rank correlation coefficient

\section{Acknowledgements}

Not applicable

\section{Authors' contributions}

$\mathrm{SB}$ and $\mathrm{FÖ}$ researched literature and conceived the study. SB, NE, MÖ, and FÖ were involved in protocol development, gaining ethical approval, patient recruitment, and data analysis. SB and NE wrote the first draft of the manuscript. All authors reviewed and edited the manuscript and approved the final version of the manuscript.

\section{Funding}

The authors would like to thank Ege University Scientific Research Projects Coordination Unit for their financial support to this project (TGA-2019-20857)

\section{Availability of data and materials}

Not applicable

\section{Ethics approval and consent to participate}

The study protocol was approved by the ethics committee of Ege University (No: 19-7 T/63). All of the assessments were conducted in accordance with the Helsinki declaration. All cases provided a written informed consent approved by the ethics committee.
Consent for publication

Not applicable

\section{Competing interests}

The authors declare that they have no competing interests

\section{Author details}

${ }^{1}$ Faculty of Health Sciences, Physiotherapy and Rehabilitation Department, Ege University, Ege Üniversitesi Sağlık Bilimleri Fakültesi 35100 Bornova, İmir, Turkey. ${ }^{2}$ Köyceğiz Vocational School of Health Services, Elderly Care Department, Muğla Sıtkı Koçman University, Ulucami Mah. Atatürk Bulvarı No:107/2, 48800 Köyceğiz, Muğla, Turkey

Received: 2 November 2020 Accepted: 26 January 2021

Published online: 04 March 2021

\section{References}

1. Yeagerman SE, Cross MB, Positano R, Doyle SM. Evaluation and treatment of symptomatic pes planus. Curr Opin Pediatr. 2011;23(1):60-7.

2. Gün K, SaridoĞan M, Uysal Ö. Pes Planus Tanısında Ayak İi ve Radyografik Ölçüm Yöntemlerinin Korelasyonu. Turk J Phys Med Rehabil. 2012;58(4):283-7.

3. Bhoir T, Anap DB, Diwate A. Prevalence of flat foot among 18-25 years old physiotherapy students: cross sectional study. Indian J Basic Appl Med Res. 2014;3(4):272-8.

4. McDonald KA, Stearne SM, Alderson JA, North I, Pires NJ, Rubenson J. The role of arch compression and metatarsophalangeal joint dynamics in modulating plantar fascia strain in running. PLoS One. 2016;11(4):1-16.

5. Kamiya T, Uchiyama E, Watanabe K, Suzuki D, Fujimiya M, Yamashita T. Dynamic effect of the tibialis posterior muscle on the arch of the foot during cyclic axial loading. Clin Biomech. 2012;27(9):962-6.

6. Ness ME, Long J, Marks R, Harris G. Foot and ankle kinematics in patients with posterior tibial tendon dysfunction. Gait Posture. 2008;27(2):331-9.

7. Bakırhan S, Angın S, Karatosun V, Ünver B, Günal I. Physical performance parameters during standing up in patients with unilateral and bilateral total knee arthroplasty. Acta Orthop Traumatol Turc. 2012;46(5):367-72.

8. Kim J-A, Lim O-B, Yi C-H. Difference in static and dynamic stability between flexible flatfeet and neutral feet. Gait Posture. 2015:41(2):546-50

9. Herring J. Tachdjian's pediatric orthopaedics. Philadelphia, London, New York, St Louis, Sydney, Toronto: WB Saunders; 2002.

10. Spörndly-Nees S, Dåsberg B, Nielsen RO, Boesen MI, Langberg H. The navicular position test-a reliable measure of the navicular bone position during rest and loading. Int J Sports Phys Ther. 2011;6(3):199-205.

11. Trudelle-Jackson E, Jackson AW, Frankowski CM, Long KM, Meske NB. Interdevice reliability and validity assessment of the Nicholas hand-held dynamometer. J Orthop Sports Phys Ther. 1994;20(6):302-6.

12. Hislop H, Avers D, Brown M. Daniels and Worthingham's muscle testing-ebook: techniques of manual examination and performance testing. Amsterdam: Elsevier Health Sciences; 2013.

13. Podsiadlo D, Richardson S. The timed "Up \& Go": a test of basic functional mobility for frail elderly persons. J Am Geriatr Soc. 1991:39(2):142-8.

14. Whitney SL, Wrisley DM, Marchetti GF, Gee MA, Redfern MS, Furman JM. Clinical measurement of sit-to-stand performance in people with balance disorders: validity of data for the five-times-sit-to-stand test. Phys Ther. 2005; 85(10):1034-45

15. Lindholm B, Nilsson MH, Hansson O, Hagell P. The clinical significance of 10-m walk test standardizations in Parkinson's disease. J Neurol. 2018;265(8): 1829-35.

16. Streiner DL, Norman GR, Cairney J. Health measurement scales: a practical guide to their development and use. New York: Oxford University Press; 2015

17. Faul F, Erdfelder E, Lang A-G, Buchner A. G* Power 3: a flexible statistical power analysis program for the social, behavioral, and biomedical sciences. Behav Res Methods. 2007:39(2):175-91.

18. Faul F, Erdfelder E, Buchner A, Lang A-G. Statistical power analyses using $G^{*}$ Power 3.1: tests for correlation and regression analyses. Behav Res Methods. 2009:41(4):1149-60.

19. KIzIlcı H, Erbahçeci F. Pes planus olan ve olmayan erkeklerde fiziksel uygunluğun değerlendirilmesi. Fizyoterapi Rehabil. 2016;27(2):25-33. 
20. Butler RJ, Hillstrom H, Song J, Richards CJ, Davis IS. Arch height index measurement system: establishment of reliability and normative values. J Am Podiatr Med Assoc. 2008;98(2):102-6.

21. Williams lii DS, McClay IS, Hamill J. Arch structure and injury patterns in runners. Clin Biomech. 2001;16(4):341-7.

22. Payne KA, Berg K, Latin RW. Ankle injuries and ankle strength, flexibility, and proprioception in college basketball players. J Athl Train. 1997;32(3):221-5.

23. Zhao X, Tsujimoto T, Kim B, Tanaka K. Association of arch height with ankle muscle strength and physical performance in adult men. Biol Sport. 2017; 34(2):119-26.

24. Aydog ST, Özçakar L, Tetik O, Demirel HA, Hascelik Z, Doral MN. Relation between foot arch index and ankle strength in elite gymnasts: a preliminary study. Br J Sports Med. 2005;39(3):1-3.

25. Vernazza-Martin S, Martin N, Le Pellec-Muller A, Tricon V, Massion J. Kinematic synergy adaptation to an unstable support surface and equilibrium maintenance during forward trunk movement. Exp Brain Res. 2006;173(1):62-78.

26. Telfer S, Abbott M, Steultjens M, Rafferty D, Woodburn J. Dose-response effects of customised foot orthoses on lower limb muscle activity and plantar pressures in pronated foot type. Gait Posture. 2013;38(3):443-9.

27. Mizner RL, Snyder-Mackler L. Altered loading during walking and sit-tostand is affected by quadriceps weakness after total knee arthroplasty. J Orthop Res. 2005;23(5):1083-90

28. Lin C-J, Lai K-A, Kuan T-S, Chou Y-L. Correlating factors and clinica significance of flexible flatfoot in preschool children. J Pediatr Orthop. 2001; 21(3):378-82.

29. Oda A. An experimental study on the effects of a flatfoot on physical motor performances: the relations of conditions evaluated from medial longitudinal arch to foot strength and physically functional motor capacities. Sendai College. 2004;5:139-48.

30. Buldt AK, Allan JJ, Landorf KB, Menz HB. The relationship between foot posture and plantar pressure during walking in adults: a systematic review. Gait Posture. 2018;62:56-67.

31. Segal A, Rohr E, Orendurff M, Shofer J, O'Brien M, Sangeorzan B. The effect of walking speed on peak plantar pressure. Foot Ankle Int. 2004; 25(12):926-33

32. Rosenbaum D, Hautmann S, Gold M, Claes L. Effects of walking speed on plantar pressure patterns and hindfoot angular motion. Gait Posture. 1994; 2(3):191-7.

\section{Publisher's Note}

Springer Nature remains neutral with regard to jurisdictional claims in published maps and institutional affiliations.

\section{Submit your manuscript to a SpringerOpen ${ }^{\circ}$ journal and benefit from:}

- Convenient online submission

- Rigorous peer review

- Open access: articles freely available online

High visibility within the field

- Retaining the copyright to your article

Submit your next manuscript at $\boldsymbol{\nabla}$ springeropen.com 